\title{
First Principles Calculations on Oxide-Based Heterogeneous Catalysts and Photocatalysts: Problems and Advances
}

\author{
Gianfranco Pacchioni
}

Received: 15 September 2014/ Accepted: 30 September 2014/Published online: 12 October 2014

(C) Springer Science+Business Media New York 2014

\begin{abstract}
Density functional theory (DFT) has become an essential complement of experiments to interpret, rationalize, and understand structures, spectroscopic data, microscopy analyses, etc. of relevance in heterogeneous catalysis and photocatalysis. However, one of the major goals of theory in catalysis remains the prediction of reaction enthalpies and entropies, of transition state structures, and the identification of reaction mechanisms. While accurate theoretical methods are currently available to study the thermochemistry of molecular systems, this is much less so when reactions involve solid surfaces and in particular oxide materials. The problem stems from the approximate nature of the exchange-correlation functionals used in all DFT approaches. Attempts to improve the accuracy of reaction energies is at the core of the efforts made in the past 30 years to generate new functionals. In this review we will discuss some recent advances in the theoretical description of oxides and their surfaces in heterogeneous catalysis and photocatalysis. In particular, we will focus on two problems: (1) the determination of an oxide band gap and the proper alignment of the occupied and unoccupied levels with respect to the vacuum level, an aspect relevant for the red-ox properties of the material, and (2) the calculation of reaction energies at oxide surfaces. To this end, we will discuss and comment on the performance of current implementations of exchange-correlation functionals (standard GGA, GGA+U, hybrid functionals, meta-GGA, etc.) or alternative approaches (like the quasiparticle $G W$ method), in predicting band
\end{abstract}

G. Pacchioni $(\square)$

Dipartimento di Scienza dei Materiali, Università di Milano-Bicocca, via R. Cozzi 53, 20125 Milan, Italy e-mail: gianfranco.pacchioni@unimib.it alignment and chemical reactivity at oxide surfaces. The role of dispersion forces will be also briefly discussed.

Keywords Band gap - Band alignment - Reaction energies · DFT · Hybrid functionals

\section{Introduction}

The description of chemical reactions occurring at the surface of a catalyst is at the center of the efforts of many theoretical chemists and physicists. Catalysis is and will remain one of the central sciences for a more sustainable approach to our societal demands [1, 2]. The modeling and theoretical description of processes occurring at the surface of a solid catalyst are expected to increase in importance also due to the increasing computational power. To this end, new methods and approaches are continuously being developed and applied to problems related to heterogeneous catalysis by oxide surfaces $[3,4]$. While theoretical chemists have developed over the past 50 years a wide set of computational approaches to study with extremely high accuracy molecular reactions (from configuration interaction [5] to coupled cluster [6] methods and quantum Monte Carlo [7] ), when one deals with reactions occurring at solid surfaces the method of choice becomes density functional theory (DFT). In the past three decades there has been a formidable advance in the computational description of adsorption and reaction at metal, semiconductor, and insulator surfaces with these methods [8, 9]. This is well demonstrated by the high number of joint theoreticalexperimental studies that are published nowadays on this topic. In this respect, DFT has become an essential tool to interpret, rationalize, and understand several characterization techniques, from vibrational spectra to photoemission 
measurements, from magnetic interactions (NMR and EPR) to absorption and emission spectroscopies, from scanning tunneling microscopy (STM) and atomic force microscopy (AFM) images to crystallographic data, etc.

Catalysis deals with the transformation of substances into other chemical entities. The role of the catalyst is to change the reaction mechanism and to lower the energy barriers involved in the transition states. One of the main tasks for theory is therefore to compute and predict reaction enthalpies and entropies, transition state structures, and to identify reaction mechanisms. All these properties rely on the accurate calculation of the total energies of reactants, intermediates and products. The small differences between very large total energies provide the quantities of interest for chemical reactivity, the reaction energies. It turns out that these are probably the most difficult quantities to compute with good level of reliability with the presently available computational tools. While most of the other properties, structures, spectroscopic values, etc. can be obtained with a good level of confidence, reaction energies remain difficult to compute accurately as rather different values are obtained with different computational setups [8]. This problem has a clear origin which is the approximate nature of the exchange-correlation functional used in all DFT approaches. Attempts to improve the accuracy of reaction energies is at the core of most of the efforts made in the past 30 years to improve the formulation of the currently used forms of the DFT approach [8].

The problem of describing catalysts based on oxide materials can be summarized in two major issues: on one side one needs an acceptable description of the electronic structure of the oxide, of the energy of its defects and localized states, and of the position of the valence and conduction band edges; on the other side an accurate description of reaction energies and energy barriers is required in order to obtain a reliable prediction of the thermodynamic and kinetic aspects of the reaction. Of course, the second aspect is related to the first one, as only an appropriate description of the electronic structure of the active surface can produce a realistic representation of its chemical reactivity.

In what follows we will discuss some of the recent advances in this field making use of some illustrative examples. In this respect, the review is clearly not comprehensive. We will discuss two main problems that are encountered when surface chemical reactions involve oxide supports: the determination of the band edges (hence of the band gap) of the oxide phase and of the defect states in the gap, as these quantities affect directly the red-ox properties of the material, and the calculation of the adsorption energies and reaction barriers at the oxide surface.

\section{Current Approaches to DFT Calculations}

As we mentioned above, DFT is the most widely used tool for the calculation of adsorption and reactions on solids surfaces, including oxides. Standard implementations of DFT are based on the Kohn-Sham equations [10] and on the use the local density or of the generalized gradient approximation (LDA and GGA, respectively) for the exchange-correlation functional. Various types of GGA functionals are available [11] like PW91 [12], PBE [13], and RPBE [14]. It is well known that LDA strongly overestimates bond strengths and adsorption energies, while GGA functionals are in much better agreement with experimental data. Other problems are inherent to the use of LDA and GGA, like for instance the underestimation of band gaps, the tendency to favor electron delocalization, and the accuracy of energy barriers for chemical reactions. These deficiencies are a consequence of the approximations present in the formulation of the exchange-correlation functional and of the lack of exact cancellation of the Coulomb self-interaction energy, an effect which is known to favor solutions with delocalization of the spin density. It is also responsible for a systematic underestimation of energy barriers [15] an effect that has also been observed for surface reactions [16].

This problem is well known among DFT practitioners, and a practical way to improve the description has been proposed about 20 years ago by Becke who suggested to use a portion of the exact Fock exchange in the exchange functional in order to minimize the self-interaction error [17]. This was the first example of the so-called hybrid functionals, and the functional is known as B3LYP, where B stays for Becke exchange functional, LYP for the correlation functional of Lee, Yang and Parr, and 3 is the number of parameters fitted to reproduce the thermochemistry of a given set of molecules. This approach turned out to be very successful and become rapidly of common use in the quantum chemistry community. So far, the original paper by Becke has received more than 45.000 citations! One should notice that very similar results are obtained when the Becke exchange is combined with other correlation parts, as in the B3PW functional [18]. Hybrid functionals have then been expanded and improved. This is the case of the PBE0 [19-21], and most recently HSE [22, 23] functionals. A characteristic of all hybrid functionals is the $\alpha$ parameter that determines the portion of exact exchange entering in the formulation of the exchangecorrelation functionals. In B3LYP, PBE0, and HSE $\alpha$ is set to $0.20,0.25$, and 0.25 , respectively. The range separated HSE functional has also an adjustable parameter controlling the short-range interaction.

The application of such functionals to periodic systems has been restricted for about a decade to codes based on 
local Gaussian basis sets like the CRYSTAL code [24]. In recent years, also the community of solid state physicists started to use hybrid functionals after these have been implemented in plane wave codes [25-27]. However, the computational effort required to evaluate the Fock exchange under periodic boundary conditions using plane waves is about two orders of magnitude larger than for a normal GGA calculation, still limiting the use of this approach. This problem does not exist if one uses local atomic orbital basis sets; in this case the cost of an hybrid functional calculation is comparable to that of a GGA calculation.

The hybrid functionals are usually more accurate than GGAs for lattice constants, atomization energies, energy gaps, etc. of inorganic solids and molecular systems (not for metals, however). For example, the use of B3LYP, PBE0 and HSE, reduces the overbinding of $\mathrm{O}_{2}$ from 3.12/ $3.10 \mathrm{eV} /$ atom (PBE/PW91) to 2.60 (B3LYP), 2.69 (PBE0), and 2.71 (HSE) eV/atom [28, 29]; these three latter values compare very well with the experimental value of $2.59 \mathrm{eV} /$ atom, corrected for the zero point effect [30]. This indicates that accurate reactions energies involving oxygen and oxides can only be expected when hybrid functionals are used.

A more pragmatic approach to describe metal oxide systems containing electrons in partially filled $d$ or $f$ states, which are localized on particular metal atoms, is the socalled DFT $+U$ approach. In the spirit of the Hubbard model [31-33], Anisimov and co-workers [34] initially proposed it as an extension of the LDA approach. In practice, in these DFT(LDA/GGA) $+U$ approaches one identifies a set of atomic-like orbitals that are treated with an orbital-dependent potential and an associated screened on-site Coulomb and exchange interaction parameters, $U$ and $J$, respectively [35]. In conventional DFT $+U$, the parameters are fixed for a given set of orbitals, and are the same for all atoms of the same type. How to chose the atomic-like orbitals and the optimal effective $U$ parameter $\left(\mathrm{U}_{\text {eff }}=U-J\right)$-hereafter referred to simply as $U$-is a delicate issue.

In this respect, $\mathrm{Hu}$ and Metiu [36] have proposed that if one is interested in the redox properties of an oxide like $\mathrm{TiO}_{2}$, the $U$ parameter should not be chosen in order to properly reproduce the band gap (this procedure usually results in high values of the $U$ term) but rather use $\mathrm{PBE}+U$ or PW91 $+U$ approaches with a $U$ value that provides a good estimate for the energy of reduction of $\mathrm{TiO}_{2}$ to $\mathrm{Ti}_{2} \mathrm{O}_{3}$. This aspect will be further discussed below in connection to some comparative studies on the reactivity of reducible oxides with various computational methods. In general, the use of DFT $+U$ is an option when the cost of running hybrid functional calculations is beyond the available computational resources (also considering that modeling of catalysis requires performing very large number of calculations).

Another important aspect, in particular for the study of molecules adsorbed on oxide surfaces, is that DFT/ DFT $+U$ with LDA or GGAs as well as hybrid-DFT do not properly account for van der Waals (vdW) dispersive interactions. In recent years, various approaches which account for vdW forces within the framework of DFT have been proposed (see Ref. [37] and references therein), and their use has considerably improved the description of molecular chemisorption. These approaches can be broadly divided into four categories: (1) methods where nonlocal correlation is either computed explicitly or integrated with traditional exchange-correlation functionals [38]; (2) exchange-correlation functionals parametrized for data sets that include non-covalently interacting systems [3941]; (3) addition of effective atom-centered nonlocal potentials [42]; and (4) addition to existing exchangecorrelation functionals of pairwise corrections [43-45].

A recent advance in the field of exchange-correlation functionals is represented by the meta-GGA functionals [46-48]. A meta-GGA DFT functional in its original form includes the second derivative of the electron density (the Laplacian). This is a natural development after the GGA, that includes only the density and its first derivative in the exchange-correlation potential. Nowadays a meta-GGA functional is more typically referred to one that includes a dependence on the kinetic energy density, i.e. on the Laplacian of the orbitals. To this family belong the M06 suite of functionals which are a set of meta-hybrid GGA DFT functionals [46-48]. They are constructed with empirical fitting of their parameters. The family includes the functionals M06-L, M06, M06-2X and M06-HF, with a different amount of exact exchange on each one. M06-L is fully local without Hartree-Fock (HF) exchange (thus it cannot be considered hybrid), M06 has $27 \%$ of $\mathrm{HF}$ exchange, M06-2X 54 and M06-HF $100 \%$. Each functional is better suited for a give class of problems. For instance, M06-L is more suited for transition metals and organometallic compounds; M06-HF is useful for cases where the self-interaction gives rise to pathological problems.

\section{The Band Gap and Band Alignment Problem}

In the study of catalytic systems the problem of the description of the band gap of an oxide material is a central one. The positions of the top of the valence band (VB) and of the bottom of the conduction band (CB) in the solid determine the red-ox properties of the oxide, as these are the electronic levels involved in charge transfer from or to the catalyst, respectively. In DFT calculations, the Kohn- 
Sham (KS) band gap is defined as the difference between the eigenvalues of the $\mathrm{CB}$ minimum (CBM) and the VB maximum (VBM). Notice that the KS band gap is only a crude approximation to the real band gap which is the result of an electronic excitation and involves, therefore, an excited state. Nevertheless, the KS band gap provides a first simple way to estimate the accuracy of the approach used.

An important aspect to mention when KS band gaps are compared to experiment is the distinction between the optical and the fundamental (or electronic) gap. This basically goes back to a distinction between different methods of measuring the energy gap. The optical gap consists in measuring the optical absorption as a function of wavelength and is equivalent to use an excitation spectroscopy: the charge state of the material does not change during the process. The fundamental band gap, on the contrary, is obtained by measuring the position of the VB and CB via electron spectroscopy, direct photoemission for the VB and inverse photoemission for the CB. Sometimes, using STM one can measure both, but this method is only possible with conducting supports like thin oxide films on metals [49]. Therefore, in common measurements of the fundamental band gap an extra electron is either injected into or taken out of the solid during the process. In order to be able to get that extra electron in, one has to overcome the Coulomb repulsion caused by all the other electrons. For this reason, the fundamental or electronic gap is usually larger than the optical gap. In solids with rather delocalized states, this effect is small, but in systems with high spatial localization of the valence and conduction states, as in several oxides, it can be large. In a photocatalyst the only important value is the optical gap, because one excites by photons and then separate electrons and holes. In a device like a light-emitting diode, however, one needs to apply a bias corresponding to the electronic gap, because one first has to inject electrons and holes into the system before they recombine to emit a photon. When one wants to compare computed and experimental band gaps one should not forget to mention which kind of gap has been measured. Dealing with insulators there is an additional problem, i.e. the formation of excitons when photons are adsorbed, and polarons when electrons are injected or holes are created.

There is no reason to expect that the HOMO-LUMO gap of the Kohn-Sham orbitals would have a physical meaning; it has to be considered as an empirical attempt that allows a rapid screening of the validity of the approach. In this spirit it is discussed below.

The "band-gap problem" in DFT is associated with the widely employed (semi)local approximations to the exchange-correlation energy. As we mentioned in $\S 2$, a practical and efficient solution to the problem that allows to

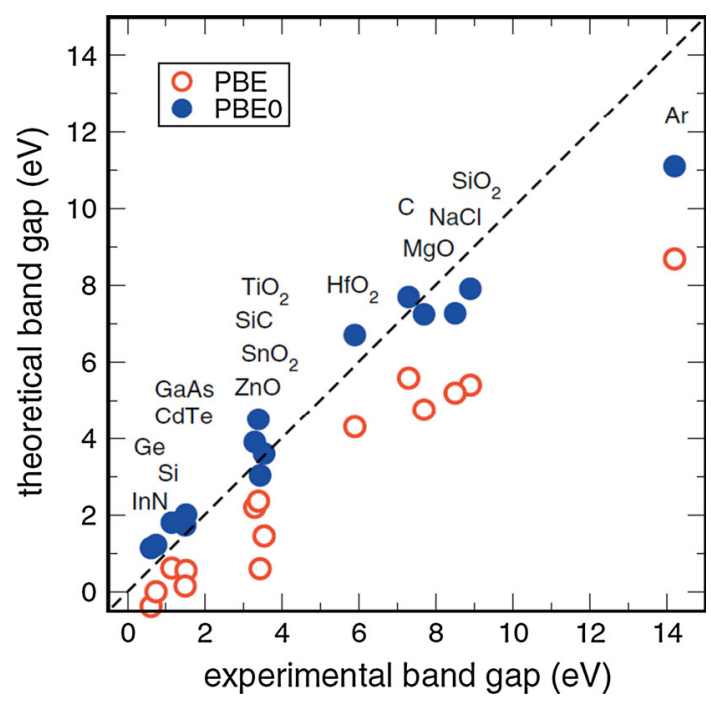

Fig. 1 Calculated versus measured single-particle KS band gaps for 15 different materials. PBE: open circles, PBE0: filled circles. Reproduced with permission from Ref. [50]

treat systems of interest is the use of hybrid functionals. This is well illustrated by a direct comparison of experimental and theoretical KS band gaps for a wide series of semiconductors and insulators as obtained with the semilocal PBE and the hybrid PBE0 functionals, Fig. 1. While significant deviations still exist between experiment and theory at the PBE0 level, the improvement with respect to the standard PBE approach is apparent [50].

The use of semilocal functionals, such as PBE, not only results in too small band gaps, but also leads to an incorrect positioning of the VB and CB edges and of the energy levels associated to defect states (intrinsic or extrinsic defects) in the band gap. Since impurity atoms introduced on purpose or by simple contamination or intrinsic defects (e.g. vacancies) can have energy levels at positions similar to the HOMO and LUMO levels of adsorbed species, an incorrect description of their position can lead to ambiguous predictions on the chemical reactivity and on the charge transfer from the oxide surface to the adsorbed species or vice versa.

The correct description of these phenomena ultimately depends on the positions of the band-edge levels. In fact, the position of defect levels can be determined reliably once the host band edges are correctly aligned with respect to an adopted reference or to the vacuum level. Many-body perturbation theory in the $G W$ approximation can be used to determine band structures, and can be considered as a reference method for electronic structure calculations of solids (in a similar was as full CI [51] or coupled cluster [52] calculations represent a benchmark of other quantum chemical approaches where various approximations are 


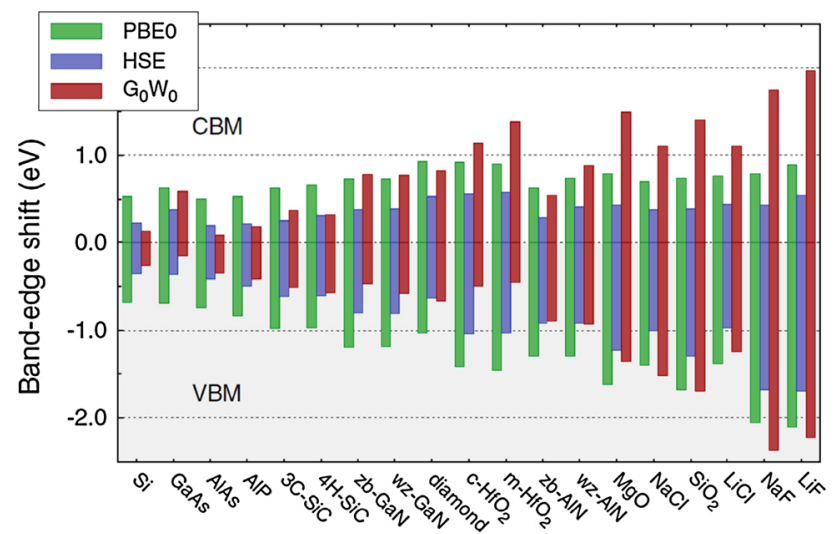

Fig. 2 The valence band maximum (VBM) and conduction band minimum (CBM) levels of semiconductors and insulators as obtained with hybrid-functionals (PBE0 and HSE) and $G_{0} W_{0}$ schemes. The $\mathrm{VBM}$ and the $\mathrm{CBM}$ are given as shifts, $\Delta E_{\mathrm{VBM}}$ and $\Delta E_{\mathrm{CBM}}$, with respect to the corresponding PBE levels. Green: PBE0; Blue: HSE; Red: $G_{0} W_{0}$. Reproduced with permission from Ref. [55]

included). Notice that GW represents a theoretical benchmark in the sense that it provides a solution at higher theoretical level than other approaches; still, also in GW calculations there are several approximations and the real benchmark should be done with clean experimental data (provided that they are available). In the $G W$ approach the electron self-energy is given by the product of the Green's function $G$ and of the screened Coulomb interaction $W$. The quasiparticle energies that are solutions of the Dyson equation give the ionization potentials (IPs) and electron affinities (EAs), and hence the fundamental IP-EA gap of the system. Using GW, one can calculate a band gap directly comparable to that obtained from photoemission/ inverse photoemission experiments. Solving the BetheSalpeter equation (BSE) [53,54] subsequent to the GW calculation accounts for electron-hole interactions, and can be used to calculate optical spectra of materials. The $G W$ approximation can also be used to determine band-offsets and defect states, with the major drawback that it has an extremely high computational cost. This limits the use of $G W$ to simple systems (the same holds true for full CI or coupled cluster calculations in molecular chemistry). Furthermore, due to their numerical cost, $G W$ calculations are mostly performed perturbatively (one-shot $G_{0} W_{0}$ ) on a set of single particle orbitals and eigenvalues obtained from a preceding DFT calculation. As an alternative, one can iterate the Dyson equation to self-consistency. We will come back to this point below, when we will discuss the band alignment in $\mathrm{TiO}_{2} / \mathrm{CH}_{3} \mathrm{OH}$ interfaces.

In a recent work, Chen and Pasquarello [55] systematically investigated the band-edge levels in a variety of materials as obtained within the most common hybrid functional schemes and compared them with those obtained within the $G W$ approximation. The materials considered ( $\mathrm{Si}$, GaAs, AlAs, AlP, GaN, SiC, diamond, AlN, $\mathrm{HfO}_{2}, \mathrm{MgO}, \mathrm{SiO}_{2}, \mathrm{LiCl}, \mathrm{NaF}$, and $\mathrm{LiF}$ ) span a large interval of band gaps and show bonding characteristics ranging from purely covalent to highly ionic. Figure 2 reports the CBM and the VBM expressed as band-edge shifts with respect to corresponding levels obtained at the PBE level. In hybrid-functional calculations, the shift in band-edge is typical of the functional but an additional variable is represented by the value chosen for the mixing parameter $\alpha$ which in principle can be "tuned" to fit experimental or more accurate results. Staying with the standard formulation of hybrid functionals, compared to PBE0, HSE systematically reduces the gap by shifting in a symmetric way the CBM and the VBM by 0.3 to $0.4 \mathrm{eV}$. By increasing the ionicity of the material, the downward shift of the VBM gradually becomes more pronounced than the upward shift of the CBM, Fig. 2.

The results indicate that, in general, the band-edge positions obtained with hybrid functionals do not coincide with those derived from $G W$ calculations. The agreement is very good for $s p$-bonded materials with band edges coinciding within $0.1 \mathrm{eV}$ when $\mathrm{HSE}$ and $G_{0} W_{0}$ results are compared. On the contrary, the presence of more contracted $d$ orbitals or the increase of the ionicity lead to deviations up to $1 \mathrm{eV}$. The conclusion is that while hybrid functionals considerably improve the description with respect to standard GGA calculations, the band edge positions might change significantly, in particular for systems with very high band gap including some oxides like $\mathrm{MgO}$ and $\mathrm{SiO}_{2}$ [55].

In a subsequent paper, Chen and Pasquarello [56] have proposed a different approach to the use of hybrid functionals aimed, beyond the KS band gap determination, to obtain a correct position of defect states in the gap from transition energy levels. Transition energy levels represent a useful tool to estimate excitation and emission energies in defective semiconductors [57-59]. They allow to locate the position of the electronic levels associated to the defects in the band gap of the material, and to go beyond the use of one-electron KS eigenvalues. The definition of transition energy level ( $\varepsilon$ ) is "the Fermi level at which two different charge states ( $\mathrm{q}$ and $\left.\mathrm{q}^{\prime}=\mathrm{q}+1 \mathrm{e}^{-}\right)$of a defect (D) have the same energy". To determine the value of $\varepsilon\left(\mathrm{q} / \mathrm{q}^{\prime}\right)$, one should start from the formation energies of the defect $\mathrm{D}$ in the charge state $q, E_{D}^{\text {form }}(q)$, and $q^{\prime}, E_{D}^{\text {form }}\left(q^{\prime}\right)$, defined with respect to the energy of the bulk host, $\mathrm{E}_{\mathrm{H}}$, the chemical potential of the species involved in creating the defect, and the Fermi level, $\mathrm{E}_{\mathrm{F}}$ :

$$
\begin{aligned}
\mathrm{E}_{\mathrm{D}}^{\text {form }}\left(\mathrm{q} \text { or } \mathrm{q}^{\prime}\right)\left(\mu, \mathrm{E}_{\mathrm{F}}\right)= & \mathrm{E}_{\mathrm{D}, \mathrm{q} \text { or } \mathrm{q}^{\prime}}-\mathrm{E}_{\mathrm{H}}+\sum \mathrm{n}_{\mathrm{i}} \mu_{\mathrm{i}} \\
& +\left(\mathrm{q} \text { or } \mathrm{q}^{\prime}\right) \times\left[\mathrm{E}_{\mathrm{v}}+\mathrm{E}_{\mathrm{F}}\right]
\end{aligned}
$$



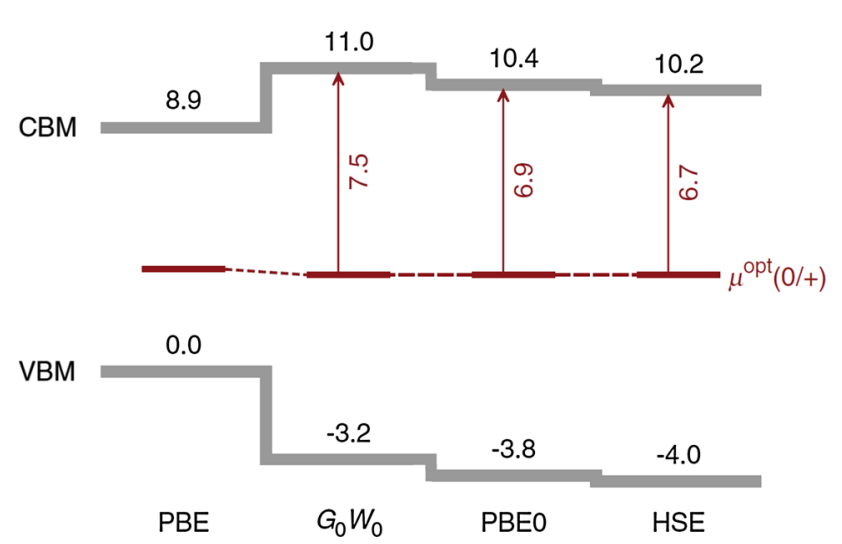

Fig. 3 Vertical charge transition levels for a fluorine vacancy $\left(\mathrm{F}^{0}\right.$ center) in LiF calculated within PBE, $G_{0} W_{0}$, PBE0, and HSE. The $G_{0} W_{0}$ and hybrid-functional calculations reproduce the experimental band gap $(14.2 \mathrm{eV})$. The energy levels are aligned with respect to the electrostatic potential of the pristine bulk. Energies are in $\mathrm{eV}$. Reproduced with permission from Ref. [56]

However, since $E_{D}^{\text {form }}(q)$ and $E_{D}^{\text {form }}\left(q^{\prime}\right)$ are equal when $E_{\mathrm{F}}=\varepsilon\left(\mathrm{q} / \mathrm{q}^{\prime}\right)$, some cancellation effects take place which lead to the following simple expression for a transition energy level:

$\varepsilon\left(q / q^{\prime}\right)=\frac{E_{D, q^{\prime}}-E_{D, q}}{q-q^{\prime}}-E_{v}$

If the top of the VB $\left(\mathrm{E}_{\mathrm{v}}\right)$ is set as the zero reference for the Fermi level, the transition energy level for an electronic transition involving one charge is simply the difference between the total energy of the defective model in the charge state $\mathrm{q}^{\prime}\left(\mathrm{E}_{\mathrm{D}, \mathrm{q}^{\prime}}\right)$ and that of the defective model in charge state $\mathrm{q}\left(\mathrm{E}_{\mathrm{D}, \mathrm{q}}\right)$. With this approach optical transition levels, $\varepsilon^{\text {opt }}\left(\mathrm{q} / \mathrm{q}^{\prime}\right)$, can be computed at fixed atomic positions (vertical transitions) and can be compared with observed features in the UPS and EELS spectra. If atomic rearrangements are taken into account, thermodynamic or adiabatic transition energy levels, $\varepsilon^{\text {therm }}\left(\mathrm{q} / \mathrm{q}^{\prime}\right)$, can be obtained:

$\varepsilon^{\text {therm }}\left(q / q^{\prime}\right)=\varepsilon^{\mathrm{opt}}\left(q / q^{\prime}\right) \pm E_{\mathrm{rel}}$

where $E_{\text {rel }}$ is the relaxation energy in absolute value, as computed from total energies difference between the charged state q' and q in their respective relaxed configuration. These values can be compared to themoluminescence and thermally stimulated current experiments.

Chen and Pasquarello [56] have shown that the $G_{0} W_{0}$ quasiparticle energy levels of localized defects are very close to the charge transition levels obtained by totalenergy differences using hybrid DFT, provided that the $\alpha$ parameter entering in the definition of the hybrid functional is chosen so as to reproduce the $G_{0} W_{0}$ quasiparticle gap. For example, the band gap in $\mathrm{SiO}_{2}$ computed at the $G_{0} W_{0}$ level is $8.77 \mathrm{eV}(8.90 \mathrm{eV}$ is the experimental value [60]). At the PBE0 level the gap is $8.21 \mathrm{eV}$, and even smaller is the prediction using the HSE functional, $7.48 \mathrm{eV}$. The fraction of Fock exchange $\alpha$ required to reproduce the $G_{0} W_{0}$ value is 0.31 (PBE0) and 0.43 (HSE), i.e. considerably higher than in the original formulation $(\alpha=0.25)$. Using these "ad hoc" hybrid functionals the charge transition levels have been determined for various systems and are very close to the defect states obtained with the $G_{0} W_{0}$ approach. This is shown, for instance, in Fig. 3 for the case of a neutral $\mathrm{F}^{0}$ center in LiF.

It is clear that in this way a different $\alpha$ is used for different materials, introducing a certain level of empiricism in the formulation of the exchange-correlation functional. On the other hand, the $\alpha$ value is obtained from "first principles", i.e. fitting the KS band gap in the hybrid calculation on the value obtained from $G_{0} W_{0}$ quasiparticle gap. This is conceptually important since often experimental measurements of band gaps are affected by accuracy problems, problems related to the exact composition of the material, formation of excitons, etc. The $G_{0} W_{0}$ calculation provides a benchmark for the electronic structure of a perfect, stoichiometric solid and as such represents a good starting point to obtain hybrid functionals with " $G_{0} W_{0}$ " accuracy at the cost of a normal hybrid DFT calculation.

A different approach to the calculation of the band gaps with hybrid functionals has been recently followed by Galli and coworkers [61]. As we have seen before, one can use $\alpha$ as an adjustable parameter to reproduce the experimental band gap of solids or the band gap obtained from $G_{0} W_{0}$ calculations. For insulating systems the screening of the long-range tail of the Coulomb interaction is proportional to the inverse of the static dielectric constant $\left(1 / \varepsilon_{\infty}\right)$. Galli and coworkers [61] have proposed a full-range, nonempirical hybrid functional where the mixing parameter $\alpha$ is determined self-consistently from the evaluation of the inverse static electronic dielectric constant $\left(1 / \varepsilon_{\infty}\right)$. This idea has been originally proposed by Alkauskas et al. [50] and by Marques et al. [62]. The dielectric constant is computed by including the full response of the electronic density to the perturbing external electric field. In this way dielectric constants, electronic gaps, and lattice constants of a broad class of solids have been determined [61]. The results are in considerably better agreement with experiments than those obtained with the semilocal PBE and the hybrid PBE0 functionals, Table 1 .

A similar approach has been followed by Conesa with the aim of properly aligning the VB and CB edges of two interfaced semiconducting oxides, specifically anatase $\mathrm{TiO}_{2}$ and $\mathrm{ZnO}$ [63]. In this approach the mixing parameter $\alpha$ has been fitted to the experimental dielectric constant $\left(\alpha=1 / \varepsilon_{\infty}\right)$ of the two materials. Next, the calculated 
Table 1 Kohn-Sham (KS) energy gaps (eV) evaluated with the dielectric-dependent hybrid functionals (adapted from Ref. [61])

\begin{tabular}{|c|c|c|c|c|c|c|}
\hline & $\begin{array}{l}\mathrm{PBE} \\
\alpha=0\end{array}$ & $\begin{array}{l}\text { PBE0 } \\
\alpha=0.25\end{array}$ & $\begin{array}{l}\text { Hybrid }^{\mathrm{a}} \\
\alpha=1 / \varepsilon_{\infty}^{\mathrm{PBE}}\end{array}$ & $\begin{array}{l}\text { Hybrid }^{\mathrm{b}} \\
\alpha=1 / \varepsilon_{\infty}^{\mathrm{PBE} 0}\end{array}$ & $\begin{array}{l}\text { Hybrid }^{\mathrm{c}} \\
\alpha=1 / \mathrm{sc}-\varepsilon_{\infty}\end{array}$ & Exp. $^{\mathrm{d}}$ \\
\hline $\mathrm{CoO}$ & 0.00 & 4.53 & - & 4.01 & 3.62 & 2.5 \\
\hline $\mathrm{TiO}_{2}$ (rutile) & 1.81 & 3.92 & 2.83 & 3.18 & 3.05 & 3.3 \\
\hline $\mathrm{WO}_{3}$ (monoc.) & 1.92 & 3.79 & 3.24 & 3.50 & 3.47 & 3.38 \\
\hline $\mathrm{ZnO}$ & 1.07 & 3.41 & 3.06 & 3.73 & 3.78 & 3.44 \\
\hline $\mathrm{MnO}$ & 1.12 & 3.87 & 2.55 & 3.66 & 3.60 & 3.9 \\
\hline $\mathrm{NiO}$ & 0.97 & 5.28 & 2.00 & 4.61 & 4.11 & 4.3 \\
\hline $\mathrm{HfO}_{2}$ & 4.32 & 6.65 & 6.38 & 6.68 & 6.68 & 5.84 \\
\hline $\mathrm{MgO}$ & 4.80 & 7.25 & 7.97 & 8.24 & 8.33 & 7.83 \\
\hline $\mathrm{H}_{2} \mathrm{O}$ & 5.57 & 8.05 & 11.19 & 11.44 & 11.71 & 10.9 \\
\hline
\end{tabular}

${ }^{a} \alpha$ is derived from the dielectric constant computed at the PBE level

${ }^{\mathrm{b}} \alpha$ is derived from the dielectric constant computed at the PBE0 level

${ }^{c} \alpha$ is derived from the dielectric constant in a self-consistent way (see Ref. [61])

d see Ref. [61]

electrostatic potential inside each slab was used as a reference for the alignment of the band-edge energies. In this way, not only the band gaps of the two oxides have been properly reproduced, but it was also possible to find that the conduction and valence bands of $\mathrm{ZnO}$ will lie ca. $0.3 \mathrm{eV}$ lower in energy than those of anatase, influencing the way in which photogenerated electrons and holes will be routed in photocatalytic systems which include interfaces between these two oxides [63].

What is probably the most detailed and extended analysis of the band alignment problem for an oxide semiconductor has been recently reported by Migani et al. for the case of methanol adsorption on rutile $\mathrm{TiO}_{2}(110)$ [64, 65]. Methanol is chosen because of the potential interest in photocatalysis. The interest of this study is that beside using a variety of computational techniques, the results have been directly compared to experimental measurements and in particular ultraviolet photoemission spectroscopy (UPS) [66] to measure the position of occupied states, two photon photoemission spectroscopy (2PP) [67] to probe the unoccupied molecular levels, and metastable impact electron spectroscopy (MIES) to measure the work function of the system [68]. On the theory side, the methods used are DFT with the PBE and the hybrid HSE functionals, and quasiparticle approaches based on $G_{0} W_{0}$ [69] and the self-consistent scQPGW [70, 71], scQPGWI [64], scQP $G W_{0}$ and scQPGW ${ }^{\mathrm{TCTC}}$ [72] methods. These latter are variants of the self-consistent $G W$ approach where a semiempirical procedure has been adopted (the reader is referred to Ref. [65] for further details; sc stays for self-consistent, QP stays for quasiparticle, TCTC stays for test charge/test charge).

Figure 4 shows the VBM and CBM energies for a bare $\mathrm{TiO}_{2}(110)$ surface from DFT (PBE and HSE), $G_{0} W_{0}$, and

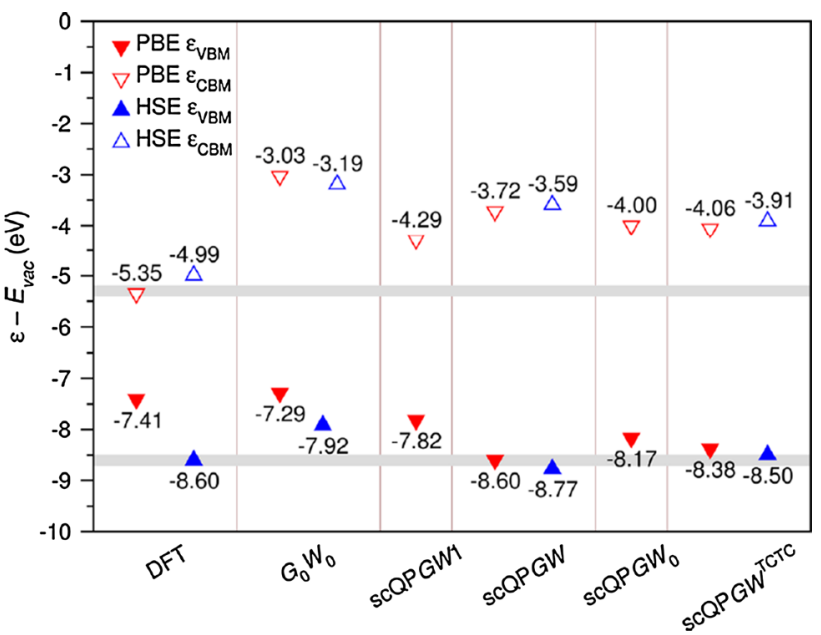

Fig. 4 Valence band maximum (VBM) and conduction band minimum $(\mathrm{CBM})$ energies $\left(\varepsilon_{\mathrm{VBM}}\right.$ and $\varepsilon_{\mathrm{CBM}}$, respectively, given in $\left.\mathrm{eV}\right)$ for a bare rutile $\mathrm{TiO}_{2}(110)$ surface, relative to the vacuum level $\mathrm{E}_{\mathrm{vac}}$ from DFT PBE, DFT HSE, $G_{0} W_{o}$, scQP $G W 1$, scQP $G W, \operatorname{scQP} G W_{o}$, and $\mathrm{scQP} G W^{\text {TCTC }}$. Gray regions denote VBM and CBM energies derived from the experimental results. Reproduced with permission from Ref. [65]

various self-consistent $G W$ approaches using PBE and HSE functionals. It is clear that all self-consistent $G W$ techniques describe the VBM level alignment consistently with the experiment. This is true also for the hybrid HSE functional. On the other hand, there is a regular overestimation of the band gap in self-consistent $G W$ methods due to a too high value of the CBM, Fig. 4. Notice that the DFT HSE approach provides an excellent estimate also of the position of the CBM, hence of the entire band gap. For the property of interest it is by far the best method. This shows, once more, the validity of this approach for practical uses. 


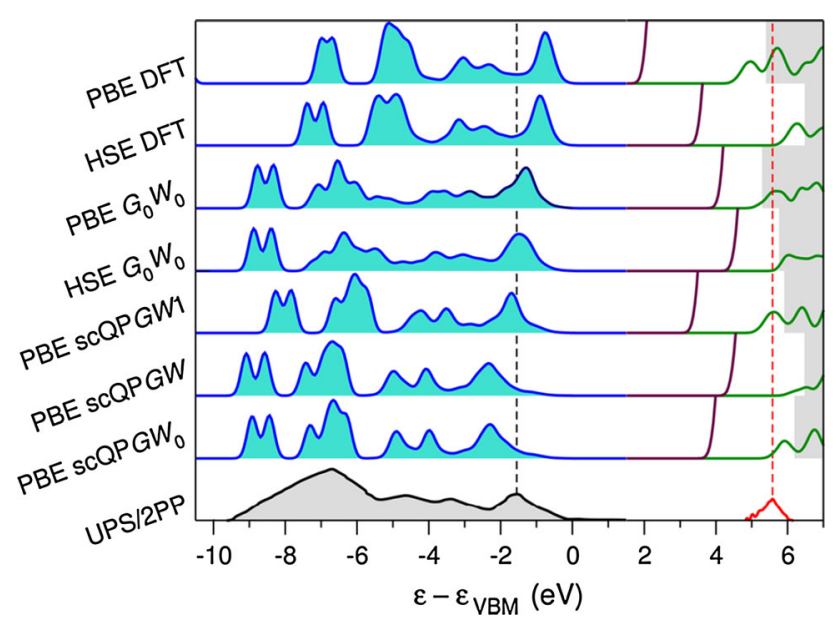

Fig. 5 Total unoccupied (maroon), $\mathrm{CH}_{3} \mathrm{OH}$ projected (blue), and wet (green) DOS computed with PBE DFT, HSE DFT, PBE $G_{0} W_{o}$, HSE $G_{0} W_{o}, \mathrm{PBE} \operatorname{scQP} G W 1, \mathrm{PBE} \operatorname{scQP} G W, \mathrm{PBE} \operatorname{scQP} G W_{0}$ for a methanol monolayer on $\mathrm{TiO}_{2}(110)$ and the experimental UPS (black) and 2PP spectra $(r e d)$. Filling denotes occupied levels. Energies are relative to the VBM $\left(\varepsilon_{\mathrm{VBM}}\right)$. Gray shaded regions denote levels above the vacuum level $\mathrm{E}_{\mathrm{vac}}$, while black/red dashed vertical lines denote the UPS/2PP highest and lowest energy peaks, respectively. Reproduced with permission from Ref. [65]

In the same way, the level alignment has been considered for the case of a monolayer of methanol adsorbed on the rutile $\mathrm{TiO}_{2}(110)$ surface, Fig. 5. To this end the $\mathrm{CH}_{3} \mathrm{OH}$ PDOS and wet DOS computed with PBE DFT, HSE DFT, PBE $G_{0} W_{0}$, HSE $G_{0} W_{0}$, PBE scQPGWl, PBE scQPGW, and PBE scQPG $W_{o}$, have been compared with UPS and 2PP spectra for a $\mathrm{CH}_{3} \mathrm{OH}$ monolayer on $\mathrm{TiO}_{2}(110)$. UPS data show that the higher energy occupied molecular levels (HOMOs) are composed of nonbonding $\mathrm{O} 2 \mathrm{p}$ orbitals of the $\mathrm{CH}_{3} \mathrm{OH}$ molecules; similarly, 2PP experiments have probed the unoccupied molecular levels for methanol on $\mathrm{TiO}_{2}(110)$. These unoccupied levels have a primarily $\sigma^{*}$ character associated with the methanol $\mathrm{C}-\mathrm{H}$ bond.

Figure 5 shows that the PBE HOMO levels are closer to the VBM than those of HSE. On the other hand, PBE overbinds the unoccupied wet levels while HSE underbinds them. $G_{O} W_{O}$ shifts the PBE energies of the empty levels up and the occupied molecular levels down, giving nearly quantitative agreement with the $2 \mathrm{PP}$ and UPS results. $G_{0} W_{O}$ shifts the HSE energies of both the unoccupied and occupied molecular levels downward, relative to the VBM, giving better agreement with the 2PP and UPS results. The PBE scQPGWI spectra shown in Fig. 5 agree even better than PBE $G_{0} W_{0}$ with the UPS and 2PP measurements. Overall, PBE $G_{0} W_{0}$ and PBE scQPGW1 provide the correct level alignment for methanol on $\mathrm{TiO}_{2}(110)$, while the other methods show some deviation from the UPS or 2PP spectra.

The work by Migani et al. [64, 65] provides an excellent benchmark of theoretical approaches to determine the band alignment in liquid/oxide interfaces. Still, the use of quasiparticle approaches is not affordable for cases of practical interest due to the prohibitive computational costs. For this purpose, one has to make use of approximate DFT functionals. Hybrid functionals, and in particular the HSE one, offer an excellent alternative to $G W$ calculations.

What remains to be investigated is if "ad hoc" hybrid functionals where the parameter $\alpha$ has been fitted to the experimental dielectric constant or to the $G W$ band gap do also provide good results in terms of surface reactivity and thermochemistry of oxide materials.

\section{The Thermochemistry Problem}

Oxides are used in catalysis either as inert supports for metal nanoparticles or directly as active catalysts. A substantial fraction of industrial catalysis is dealing with oxidation reactions or oxidative dehydrogenation processes where the catalyst is an active oxide. The most common mechanism is that described by Mars and van Krevelen [73] where an organic substrate reacts with specific sites and in particular with more reactive oxygen atoms located at low-coordinated sites at the oxide surface; an $\mathrm{O}$ atom is added to the reactant leaving behind an oxygen vacancy, thus altering the stoichiometry of the starting material. The reaction occurs under oxygen pressure, and gas-phase $\mathrm{O}_{2}$ can interact with the surface, dissociate, and fill the vacancy created in the oxidative process thus restoring the original stoichiometry and composition of the catalyst. Using isotopically labeled oxygen it has been demonstrated that the oxygen atom incorporated in the organic reactant does not come from the gas phase but directly from the oxide surface [74]. Clearly, the cost of removing an oxygen atom from the surface of the catalyst with formation of an oxygen vacancy is an essential parameter in determining both the kinetics and the thermodynamics of the reaction [75].

Given the role that oxygen release has on the surface chemistry and catalysis of oxides, it is not at all surprising that several attempts have been made to chemically modify the cost for oxygen removal from an oxide by, for instance, doping the material with heteroatoms [76]. Doping oxides by heteroatoms has been studied recently both experimentally and theoretically with the hope to produce better catalytic materials, in particular for oxidation reactions [76-78]. Clearly, the number of possibilities is extremely large, since in principle all atoms of the periodic table can be used to replace a metal cation (or the oxide anion) in an oxide. The field has been recently reviewed by McFarland and Metiu [79].

The replacement of a metal cation in an oxide of $\mathrm{M}_{\mathrm{x}} \mathrm{O}_{\mathrm{y}}$ formula with a dopant $\mathrm{D}$, opens several possibilities to 
selectively modify the electronic structure of the oxide. If the doping heteroatom $\mathrm{D}$ has the same valency of the metal cation $\mathrm{M}$ the changes are mostly related to the different size of the two cations and to the different strength of the $\mathrm{M}-\mathrm{O}$ and $\mathrm{D}-\mathrm{O}$ bonds. However, if the $\mathrm{D}$ dopant has a different number of valence electrons several possibilities exist to compensate the charge. This opens a complex scenario of defects that can more easily form and appear in the material, also as a function of the preparation conditions. This effect has been studied in detail for the case of $\mathrm{Cr}$, Mo, and $\mathrm{Nb}$-doped $\mathrm{MgO}$ and $\mathrm{CaO}$ surfaces [80-82]. The transition metal (TM) elements introduce new states in the gap of $\mathrm{CaO}$ or $\mathrm{MgO}$ that change the reactivity of the oxide surface. In particular, the TM atom can donate some of its valence electrons to other defects present in the structure or to adsorbed species like Au atoms and clusters or $\mathrm{O}_{2}$ molecules with formation of negatively charged adsorbates [80-83]. The position of the defect states in the band gap of the ionic oxide $(\mathrm{CaO}$ or $\mathrm{MgO})$ could critically depend on the level of theoretical treatment used, as discussed above. However, comparison of GGA and hybrid DFT calculations has shown unambiguously that while the use of the hybrid functional results in a larger band gap of the oxide, the relative position of the $d$ states of the TM impurity with respect to the acceptor levels of the adsorbed species do not change with the method, so that the occurrence and the direction of the charge transfer are independent of the level of treatment [84]. The absolute values of the adsorption energies, on the contrary, change significantly, showing that while the energetics of the process is critically dependent on the method used to describe the system, the physical mechanism is not [84].

The shortcomings of approximate exchange-correlation functionals become obvious when modeling complex oxides such as defective $\mathrm{CeO}_{2}$, a widely studied oxide with DFT methods [85]. In the stoichiometric material all Ce ions are in a +4 oxidation state so that their $4 \mathrm{f}$ orbitals are empty. The material belongs to the class of $\mathrm{f}^{0}$ compounds, and the only problem in the description at the DFT level is that of the band gap discussed above. No unpaired electrons are present in the ground state of the non-defective system.

The problem appears when the material is in a reduced form and $\mathrm{Ce}^{4+}$ ions trap an electron to form $\mathrm{Ce}^{3+}$. This corresponds to the formation of very localized, atom-like $4 \mathrm{f}$ orbitals whose corresponding energy levels fall in the gap of the material. The complications arise from the selfinteraction error intrinsic to semilocal functionals discussed above. The self-interaction error, being responsible for too low band gaps, also results in an underestimation of the energetic cost to occupy the $\mathrm{CB}$, and in turn can affect the energy cost for removing an oxygen atom. In particular, it can result in too low defect formation energies [86]. In
Table 2 Lattice parameters, $a_{0}$ and $c_{O}$ in $\AA$, and reaction energies, $\Delta \mathrm{E}$ in $\mathrm{kJ} / \mathrm{mol}$, for the reduction of $\mathrm{CeO}_{2}$ to $\mathrm{Ce}_{2} \mathrm{O}_{3}$ according to reactions (4a) and (4b) (adapted from Ref. [85])

\begin{tabular}{|c|c|c|c|c|c|}
\hline & \multicolumn{2}{|c|}{$\mathrm{CeO}_{2}$} & \multirow{2}{*}{$\begin{array}{l}\mathrm{Ce}_{2} \mathrm{O}_{3} \\
a_{0}\end{array}$} & \multicolumn{2}{|l|}{$\Delta \mathrm{E}$} \\
\hline & $a_{0}$ & $c_{0}$ & & $\begin{array}{l}\text { Reaction } \\
\text { (4a) }\end{array}$ & $\begin{array}{l}\text { Reaction } \\
\text { (4b) }\end{array}$ \\
\hline Exp. & 3.89 & 6.06 & 5.41 & 388 & 149 \\
\hline $\mathrm{PBE}+U(U=4.5)$ & 3.92 & 6.18 & 5.49 & 221 & -20 \\
\hline HSE & 3.87 & 6.08 & 5.40 & 305 & 53 \\
\hline PBE0 & 3.87 & 6.07 & 5.39 & 303 & 51 \\
\hline $\mathrm{HSE}+\mathrm{D}$ & 3.85 & 6.08 & 5.39 & 327 & 75 \\
\hline $\mathrm{PBE} 0+\mathrm{D}$ & 3.86 & 6.07 & 5.39 & 327 & 75 \\
\hline PBE & 3.83 & 6.08 & 5.47 & 403 & 162 \\
\hline
\end{tabular}

fact, removing oxygen corresponds to reduce the material, and the extra electrons that remain in the system can be transferred to empty $\mathrm{CB}$ states. If these states are energetically too low, the process is artificially favored.

We already mentioned that the two currently adopted approaches to circumvent the problem are DFT $+U$ and hybrid functionals. Many studies caution against inherent problems to the DFT $+U$ approach, in particular the rather strong dependence of band gap, lattice constant, etc., on the $U$ value. The problem is that different values of $U$ are required for different properties. For instance, if one wants to reproduce the electronic structure and the band gap of $\mathrm{CeO}_{2}$ one needs $U$ values between 4.5 and $6 \mathrm{eV}[87,88]$. On the contrary, in order to fit the experimentally determined adsorption energy of $\mathrm{CO}$ on $\mathrm{CeO}_{2}$ one needs to set $U=2$ [89]. A single $U$ value is not able to provide a good description of both properties.

Even more surprising are the results reported by Branda et al. [90] who found that the oxidation state of $\mathrm{Au}$ on $\mathrm{CeO}_{2}(111)$ critically depends on the value chosen for $U$. Depending on the $U$ value the lattice constant of ceria changes and with it the ability of an adsorbed Au atom to transfer electrons to the support. Therefore, different $U$ values not only result in changes in absolute adsorption energies, with a quantitative effect, but may even produce a qualitatively different description of the interaction, going from a situation where the bonding has charge transfer character to one where this is no longer present [90].

An alternative to DFT $+U$ is the use of hybrid functionals. Recently, Graciani et al. [91] have studied in detail the performance of various hybrid functionals for the description of cell parameters, band gaps, and reaction energies of ceria, Table 2. In particular, they considered two different chemical reductions of ceria:

$2 \mathrm{CeO}_{2} \rightarrow \mathrm{Ce}_{2} \mathrm{O}_{3}+1 / 2 \mathrm{O}_{2}$
$2 \mathrm{CeO}_{2}+\mathrm{H}_{2} \rightarrow \mathrm{Ce}_{2} \mathrm{O}_{3}+\mathrm{H}_{2} \mathrm{O}$ 
Table 3 Binding energy, $\Delta \mathrm{E}$ in $\mathrm{kJ} / \mathrm{mol}^{\mathrm{a}}$, of various ORR intermediates on $\mathrm{LaXO}_{3}$ surfaces at the PBE, PBE $+U$ and HSE levels

\begin{tabular}{llrrrr}
\hline & & $\Delta \mathrm{E}_{\mathrm{OH}}$ & $\Delta \mathrm{E}_{\mathrm{OO}}$ & $\Delta \mathrm{E}_{\mathrm{OOH}}$ & $\Delta \mathrm{E}_{\mathrm{O}}$ \\
\hline $\mathrm{LaMnO}_{3}$ & $\mathrm{PBE}$ & 282.5 & 63.6 & 137.4 & 362.3 \\
& $\mathrm{PBE}+U$ & 203.3 & 19.8 & 69.9 & 191.7 \\
& $\mathrm{HSE}$ & 158.9 & -15.6 & 46.5 & 123.3 \\
$\mathrm{LaFeO}_{3}$ & $\mathrm{PBE}$ & 259.2 & 47.4 & 118.1 & 333.6 \\
& PBE $+U$ & 220.7 & 19.2 & 76.6 & 205.9 \\
& $\mathrm{HSE}$ & 171.6 & -31.6 & 2.7 & 132.0 \\
$\mathrm{LaCrO}_{3}$ & $\mathrm{PBE}$ & 316.1 & 89.8 & 172.3 & 481.1 \\
& PBE $+U$ & 243.4 & 16.6 & 97.8 & 339.2 \\
& $\mathrm{HSE}$ & 179.3 & -29.4 & 65.4 & 286.8 \\
\hline
\end{tabular}

${ }^{\text {a }}$ DFT binding energy calculated as $\Delta \mathrm{E}=\mathrm{E}\left(\mathrm{LaXO}_{3}\right)+\mathrm{E}($ adsorbate $)-$ $\mathrm{E}\left(\mathrm{LaXO}_{3} /\right.$ adsorbate $)$

Both reactions involve the formation of $\mathrm{Ce}^{3+}$ ions starting from $\mathrm{Ce}^{4+}$, but the detailed mechanism is clearly different. Since DFT has problems in describing the binding energy of the $\mathrm{O}_{2}$ molecule involved in reaction (4a) (see above), the second reaction (4b) should remove this problem. This case has been discussed in detail also by Sauer and coworkers in a recent extensive review [85]. Different functionals have been applied for the study of chemical reduction of ceria, according to reactions (4a) and (4b), Table 2 [85]. At the PBE $+U$ level with $U=4.5 \mathrm{eV}$ the lattice constants of $\mathrm{CeO}_{2}$ and $\mathrm{Ce}_{2} \mathrm{O}_{3}$ are overestimated, while the bulk reduction energy of $\mathrm{CeO}_{2}$ is severely underestimated, Table 2. This reflects the low value of the $\mathrm{CB}$ in DFT $+U$. The PBE0 and HSE hybrid functionals give significantly better results but still underestimate the experimental values by about $80 \mathrm{~kJ} / \mathrm{mol}$, Table 2 (here a note of caution is necessary: the measurement of reaction energies is not without problems for solid systems). Adding a dispersion term to the hybrid functionals (PBE0 $+\mathrm{D}$ and HSE + D) improves the reduction energies by about $20 \mathrm{~kJ} /$ $\mathrm{mol}$, but the values are still far from the experimental ones. Furthermore, reaction (4b), where the reference is a water molecule instead of $\mathrm{O}_{2}$, does not improve the results compared to $1 / 2 \quad \mathrm{O}_{2}$ as reference. Surprisingly, the conventional PBE functional seems to perform well, with reaction energies that are only 15 and $13 \mathrm{~kJ} / \mathrm{mol}$ larger than the experimental values for reactions (4a) and (4b), respectively. However, according to Sauer et al. [85], this result is due to a fortuitous cancellation of errors that is seen when the energies of formation of $\mathrm{Ce}_{2} \mathrm{O}_{3}$ and $\mathrm{CeO}_{2}$ are considered.

Another example of the problematic description of chemical reactions at oxide surfaces comes from a recent study on the oxygen reduction reaction (ORR), a process relevant in electrochemical energy conversion, on the surface of perovskites [92]. Recent experiments show that perovskite transition-metal oxides can exhibit high electrocatalytic activity for ORR in alkaline electrolytes [93]. ORR on perovskites has been studied in the past using first principles approaches based on DFT [94, 95]. In general, these studies considered atomic and molecular oxygen adsorption on the $\mathrm{LaXO}_{3}$ surface (where $\mathrm{X}$ is a TM cation). However, the ORR involves a more complicated reaction pathway and various surface intermediates such as hydroxides and peroxides are involved. In a recent study Wang and Cheng [92] reported a comparative theoretical analysis of ORR activity on $\mathrm{LaXO}_{3}(\mathrm{X}=\mathrm{Mn}, \mathrm{Fe}, \mathrm{Cr})$ perovskite oxide surfaces using GGA, GGA+U, and hybrid functionals. The structures of $\mathrm{OO}, \mathrm{O}, \mathrm{HO}$, and $\mathrm{HOO}$ intermediates on the surface of $\mathrm{LaXO}_{3}$ perovskites have been optimized at the PBE, PBE $+U(U=4(\mathrm{Mn}), 4(\mathrm{Fe})$, and $3.5(\mathrm{Cr})$ ), and at the HSE levels, see Table 3 and Fig. 6.

Among the four ORR intermediates, the $\mathrm{O}$ binding energy shows the strongest dependence of the calculating method. Taking $\mathrm{LaMnO}_{3}$ as an example, the PBE binding energy is about $170 \mathrm{~kJ} / \mathrm{mol}$ larger than that of PBE $+U$, and is about $240 \mathrm{~kJ} / \mathrm{mol}$ larger than the HSE value, Table 3. For the other fragments considered the oscillations are only slightly less pronounced. For the OO adsorption even the sign of the adsorption energy changes, Table 3 . It should be noted that while the binding energies show such a strong oscillation, the nature of the bonding and the atomic charge on the fragments are basically the same with the various methods, indicating once more that DFT approaches are quite solid in terms of bond description and that the major limitation is presently the accuracy in the calculated energies. Not surprisingly, when the adsorption energies are converted into Gibbs free energies by adding entropic and zero-point energy corrections to the ORR intermediates, quite different thermodynamic results are found depending on the level of theory used [92].

The question becomes therefore which of the methods provides the better description of the ORR processes. The results calculated from the hybrid-functional method suggest that the order of ORR activity is $\mathrm{LaMnO}_{3}>\mathrm{LaCrO}_{3}>$ $\mathrm{LaFeO}_{3}$. This is in considerably better agreement with recent experimental observation in ref [93] than those from the PBE or PBE $+U$ methods. The PBE results yields similar free energy diagrams and ORR activities for $\mathrm{LaMnO}_{3}$ and $\mathrm{LaFeO}_{3}$, while the PBE $+U$ data suggest that $\mathrm{LaCrO}_{3}$ has the lowest ORR overpotential. In neither case does the obtained order of ORR activity agree with the experiment [92]. It should be mentioned that that the effect of liquid water was not taken into account in the calculations, making a direct comparison with experiments delicate.

The results discussed above for $\mathrm{CeO}_{2}$ and $\mathrm{LaXO}_{3}$ show how difficult is the calculation of reaction energies 
involving reducible transition metal or rare-earth oxides. The use of hybrid functionals remains preferable since they are consistently applied to all atomic species of a system as well as to all orbitals, contrary to DFT $+U$, where $U$ is applied to orbitals of a certain angular momentum of a particular atomic species only. Of course, hybrid functionals contain parameters that fix the amount of Fock exchange and the computational results will depend on these parameters, as we have seen above for the calculation of band gaps. In general, GGA+U provides the proper electron localization but describes energy differences only qualitatively right. Hybrid functionals are therefore a better choice and represent at the moment the best practical solution to the problem.

Of course, there are also drawbacks. One is the already mentioned high computational cost when plane wave codes are used in combination with hybrid functionals; the other is that hybrid functionals do not work so well for metallic systems, although also in this case there are differences (for instance, the HSE functional seems to perform better for metals than other hybrid functionals $[25,96])$. This problem is particularly relevant when one is interested in metal/ oxide interfaces (supported oxide films on metals, metal nanoparticles deposited on an oxide support, etc.). In this case, the use of hybrid functionals can lead to an improvement of the description of the insulating phase at the expense of a less accurate description of the metallic phase. Here the use of GGA $+U$ approaches represents a reasonable compromise, as this allows to correct the deficiencies of standard DFT for those specific atoms involved in the insulating phase, treating the rest of the metallic component at the pure GGA level.

A step forward in the search of accurate functionals for reactions at oxide surfaces is represented by the already mentioned M06-L and related meta-GGA functionals of Zhao and Truhlar [46-48] which include not only the density and its first derivative, but also the kinetic energy density. A properly constructed meta-GGA functional can detect and correct for electron over-delocalization without including non-local HFlike exchange. Indeed, the M06-L functional contains a kinetic energy density-based term in its correlation functional that exactly removes the self-interaction error for a oneelectron system. In addition, since the M06-L functional makes use of 35 adjustable parameters introduced in its construction, it should provide superior accuracy over PBE and RPBE, which contain no such empirical parameters.

The performance of M06-L functional in the reactivity of oxides has been recently discussed by Getsoian and Bell [97] who have studied the reaction of hydrogen abstraction from propene over a $\mathrm{Bi}_{2} \mathrm{Mo}_{3} \mathrm{O}_{12}$ catalyst and compared the reaction profile obtained using the meta-GGA M06-L functional, with a standard GGA functional (RPBE) and a DFT $+U$ approach (RPBE $+U$ with $U=8.6 \mathrm{eV}$ ), Fig. 7 .

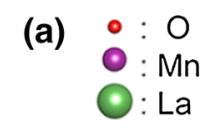
(b)

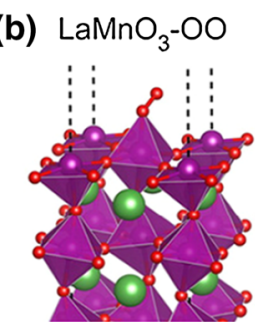

(c)
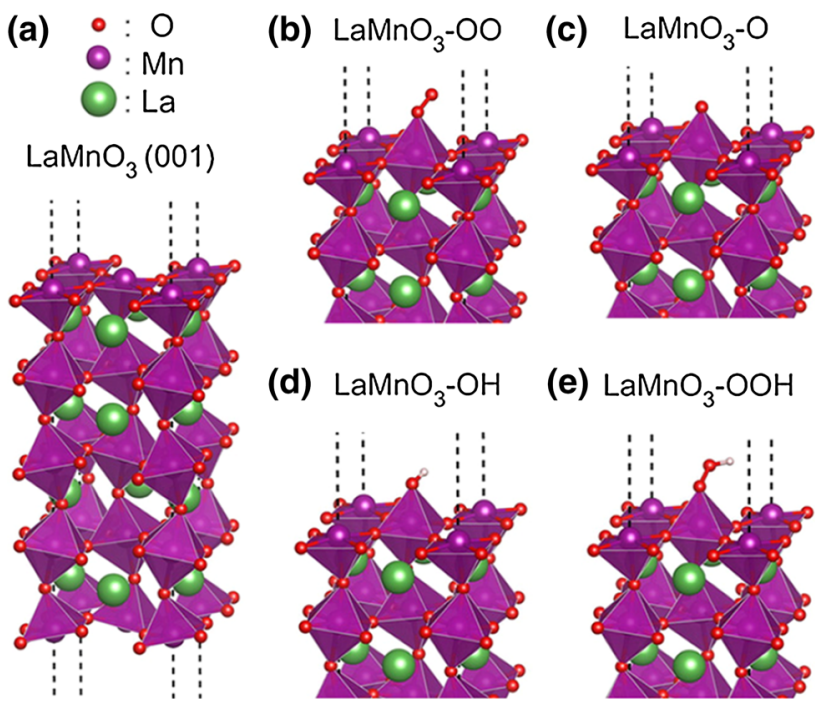

(d)

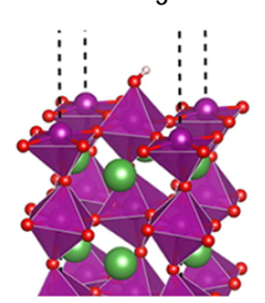

(e) $\mathrm{LaMnO}_{3}-\mathrm{OOH}$

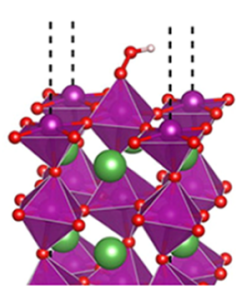

Fig. 6 Optimized geometry of (a) bare $\mathrm{LaMnO}_{3}(001)$ surface and (b-e) surfaces with adsorbed $\mathrm{OO}(\mathbf{b}), \mathrm{O}(\mathbf{c}), \mathrm{OH}(\mathbf{d})$, and $\mathrm{OOH}$ (e) intermediates. The dashed lines are the boundaries of the supercell. Reproduced with permission from Ref. [92]

Significant differences have been found both in the structure of the transition states and in the energy profiles, depending on the method used. For instance, in the M06-L reaction path the propene molecule sits over a bismuth atom while the RPBE $+U$ calculation captures essentially no dispersive interactions and thus orients the propene into a sterically uncrowded site with no specific surfaceadsorbate interactions. Also the energy barriers are very different in M06-L and RPBE, while are comparable in M06-L and RPBE $+U$, Fig. 7. However, the transition state structure predicted by the M06-L functional provides better agreement with experimental trends than that obtained with RPBE $+U$ functional. The general conclusion of Ref. [97] is that while DFT calculations carried out with the M06-L functional are computationally more expensive than those performed with DFT $+U$ (by a factor 5 ), they provide sensible results for all properties investigated, and avoid the somewhat arbitrary choice of the $U$ term in DFT $+U$.

\section{Role of Dispersion for Adsorption on Oxide Surfaces}

A brief comment on the role of dispersion for the description of thermochemistry at oxide surfaces is in order. In the past decade, many efforts have been made to include van der Waals interactions in DFT calculations [98]. These bonding contributions have been entirely neglected in DFT calculations until it was finally recognized that their role is by no means negligible (but nevertheless, very often "good agreement" with experiment has 
(a)

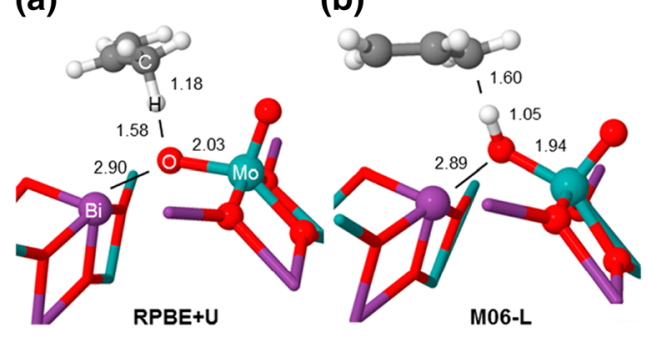

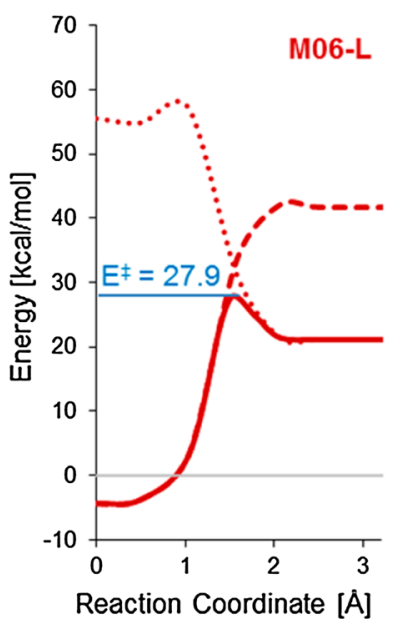
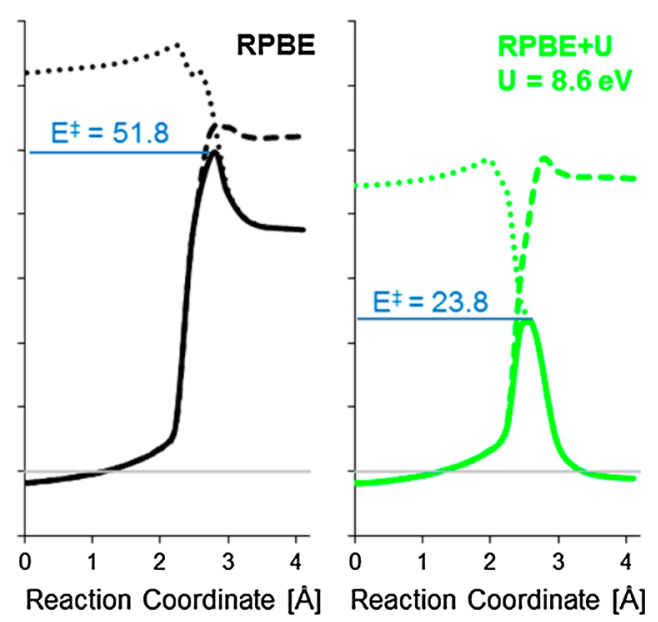

Fig. 7 Left transition states for hydrogen abstraction from propene calculated using $\mathrm{RPBE}+U(U=8.6 \mathrm{eV})$ (a) and M06-L (b). Distances are in $\AA$. Right activation barriers for hydrogen abstraction from propene over $\mathrm{Bi}_{2} \mathrm{Mo}_{3} \mathrm{O}_{12}$ calculated using M06-L, RPBE, and $\mathrm{RPBE}+U(8.6 \mathrm{eV})$. The singlet spin states are indicated by long

been claimed despite the fact that these bonding contributions were completely neglected). The role of dispersion is obvious when one is interested in molecular crystals or weakly interacting systems, but also adsorption at surfaces is significantly affected by these terms, in particular when large molecules are considered.

Oxide surfaces are no exception. An example is provided by a recent study on the adsorption of bromobenzene and aniline on the $\mathrm{Cu}_{2} \mathrm{O}(110)$ and (111) surfaces [99]. The calculations have been performed using the revPBE functional [100] and the vdW-DF approach of Lundqvist and co workers [101]. In the first case dispersion forces are not included, differently from vdW-DF. In Fig. 8 some illustrative results are reported for bromobenzene adsorbed on various sites of the two $\mathrm{Cu}_{2} \mathrm{O}$ surfaces (in Fig. $8 \mathrm{~T}-0-\mathrm{T}-120$ indicate the tilt angle of the molecule with respect to a crystallographic direction). The interaction with the (110) surface is dominated by dispersion. In fact, at the revPBE level the organic molecule is virtually unbound, while at the vdW-DF level the adsorption energy is of about $0.5 \mathrm{eV}$. Also the geometry is clearly affected with vertical distances from the surface which are reduced by up to $0.7 \AA$. Furthermore, the angle formed by the molecule with the surface is completely different in the two cases [99]. On the (111) surface, on the contrary, the interaction is stronger also due to the presence of coordinatively unsaturated $\mathrm{Cu}$ sites. The binding energy, which goes from 0.6 to $1.1 \mathrm{eV}$ at the revPBE level, increases by $0.2-0.3 \mathrm{eV}$ only when vdW forces are included and becomes $0.9-1.3 \mathrm{eV}$, depending on the orientation of the molecule. Quite relevant is the role of dispersion on the adsorption geometries: substantial dashes, the triplet spin states by short dots, and the spin-coupled reaction paths by solid lines. The zero of energy (gray line) is set by the energy of the bare slab and gas phase propene taken separately. Reproduced with permission from Ref. [97]

changes are found both in the surface-to-molecule distance and in the tilt angle upon inclusion of dispersion, Fig. 8.

These results clearly show that, not surprisingly, for chemisorption on oxide surfaces dispersion modifies the absolute values of the adsorption energies without changing the general picture; for physisorption interactions, on the contrary, a completely different picture emerges when $\mathrm{vdW}$ forces are included.

\section{Summary and Outlook}

The use of DFT studies to complement work in heterogeneous catalysis is continuously expanding. DFT calculations can be used to interpret experimental data, in particular results coming from specific spectroscopies or microscopies, or to provide data that are difficult to obtain experimentally, like for instance structures of intermediates, reaction barriers and mechanisms.

In the last decades we assisted to a continuous evolution in the field, and methods and approaches are becoming more sophisticated and complex in order to provide solid and reliable results. In 2008 we published a review paper on a similar subject with the title: "Modeling doped and defective oxides in catalysis with DFT methods: room for improvements" [102]. Since then, significant improvements have become reality. New methods and approaches have been implemented in electronic structure codes and have entered into the arsenal of computational tools available to mimic reactions and processes at oxide surfaces. 
Fig. 8 Optimized structures and adsorption energies of bromobenzene on $\mathrm{Cu}_{2} \mathrm{O}$ (110) and (111) surfaces. A negative $\Delta \mathrm{E}$ indicates a bound complex. Reproduced with permission from Ref. [99]

\begin{tabular}{|c|c|c|c|c|c|}
\hline & (110) & $(110)$ & (111) & (111) & (111) \\
\hline & T-0 & T-90 & $T_{\text {Cucus }}-90$ & $\mathbf{T}_{\text {Cucsa }} \mathbf{- 0}$ & $T_{\text {Cucsa }}-120$ \\
\hline \multicolumn{6}{|l|}{ Top view } \\
\hline \multicolumn{6}{|l|}{$\begin{array}{c}\text { revPBE } \\
\text { side view }\end{array}$} \\
\hline \multicolumn{6}{|l|}{$\begin{array}{l}\text { vdW-DF } \\
\text { side view }\end{array}$} \\
\hline $\mathrm{z}_{\mathrm{revPBE}}[\AA]$ & 4.09 & 4.10 & 3.88 & 3.98 & 3.98 \\
\hline $\mathrm{Z}_{\mathrm{vdW}-\mathrm{DF}}[\AA]$ & 3.41 & 3.80 & 3.7 & 3.3 & 3.58 \\
\hline$\left.\theta_{\text {revPRF }}\right|^{0} 1$ & -7.6 & 0.6 & 26.5 & -10.2 & -10.7 \\
\hline$\theta_{\mathrm{vdW}-\mathrm{DF}}\left[{ }^{0}\right]$ & 2.6 & 2.9 & 24.3 & 2 & -4.3 \\
\hline$\Delta \mathrm{E}_{\text {ads,revPBE }}{ }^{\text {relax }} / \mathrm{eV}$ & -0.03 & 0 & -1.1 & -0.8 & -0.58 \\
\hline$\Delta \mathrm{E}_{\mathrm{ads}, \mathrm{vdWDF}}{ }^{\text {relax }} / \mathrm{eV}$ & -0.53 & $-0.55 \mathrm{eV}$ & -1.33 & -1.1 & -0.85 \\
\hline
\end{tabular}

On the side of the description of the band gap of semiconducting and insulating oxides, two important advances have to be mentioned. The first one is that it is now generally accepted that hybrid functionals provide an efficient cure to the self-interaction problem (it has not been so for quite some time, mainly due to the success of semilocal functionals in the description of metallic systems); the consequence of this is that hybrid functionals have been implemented in several plane wave codes and their use is now widespread. The second important advance is that many-body calculations based on the $G W$ approximation have become available. Their cost is still prohibitive and $G W$ calculations cannot be used directly to study surface adsorption and reactions. However, $G W$ calculations provide in principle a well defined benchmark for the band gap of ideal, stoichiometric, non defective materials. The band gaps derived from $G W$ calculations can be used to fit the amount of exact exchange to be used in hybrid functionals. These, in turns, represent from a pragmatic point of view the best approaches for the theoretical study of band gaps in photocatalytic materials and their defect states. The cost of hybrid functionals remains very high when codes based on plane waves are used, while it is affordable with codes using atomic orbital basis sets or other localized functions. Hybrid functionals can also be parametrized using the dielectric constant of the solid to study or the theoretical $G W$ band gap. This provides good results in terms of band gap and band alignment, but the validity of this approach for surface reactions still needs to be explored.
More complex is the problem of the description of the thermochemistry and of the stability of oxide materials. Here the most important advance is probably represented by the introduction of the meta-GGA functionals. While more expensive than the hybrid functionals, these methods seem to offer a good compromise between accuracy and computational cost.

The third advance in the last decade has been the development of various methods to include dispersion forces in DFT calculations. These contributions are by no means negligible, and should be included in every study of adsorption and reactivity at oxide surfaces. Of course, the role of dispersion is minor when strong chemical bonds are formed, but is rather important in the description of how molecules interact and diffuse on a surface.

These specific advances, associated to the continuous development of electronic structure codes and to the increasing computer power, open a bright perspective for practitioners interested in modeling reactions at oxide surfaces including heterogeneous catalysis and photocatalysis. It should be stressed, however, that it is very important to be able to identify which method is the most appropriate for a given problem, and that the quality of the results critically depends on this specific capability. At the moment, there is no single universal solution for every chemical or physical problem. The choice of the most appropriate theoretical method to address a specific problem in catalysis or photocatalysis is the necessary prerequisite for a successful modeling of these complex systems. 
A final comment is in order. So far we have concentrated on the problem of properly describing the electronic structure of a solid crystalline material with well defined composition and without imperfections. Unfortunately, to model real catalytic systems one has to include many of the complexities that make a real catalyst working: presence of low coordinated sites, morphological irregularities, defects and impurities, not to talk of the dynamic aspects of the problem (under catalytic conditions the surface is not a rigid object but rather evolves and undergoes modifications depending on external parameters like temperature and pressure). Therefore, in order to model catalysis at oxide surfaces one has to be aware of the limitations inherent to the choice of regular, non-defective single crystal surfaces. Without taking into account this aspect, the understanding of catalytic systems and processes relying on accurate electronic structure calculations on incorrect models will be insufficient or even erroneous. Theory has done enormous progresses in the description of these systems, but the way towards a comprehensive description of real catalysts from first principles is still quite long.

Acknowledgments I acknowledge financial support from the following funding bodies: the European Community's Seventh Framework Programme FP7/2007-2013 under Grant Agreement No. 604307 (CASCATBEL) and the Grant Agreement No. 607417 (CATSENSE); the Italian MIUR through the FIRB Project RBAP115AYN "Oxides at the nanoscale: multifunctionality and applications"; the CARIPLO foundation for the project "Nuovi materiali fotocatalitici per la conversione di energia solare basati su eterogiunzioni"; the COST Action CM1104 "Reducible oxide chemistry, structure and functions".

\section{References}

1. Appel AM, Bercaw JE, Bocarsly AB, Dobbek H, DuBois DL, Dupuis M, Ferry JG, Fujita E, Hille R, Kenis PJA, Kerfeld CA, Morris RH, Peden CHF, Portis AR, Ragsdale SW, Rauchfuss TB, Reek JNH, Seefeldt LC, Thauer RK, Waldrop GL (2013) Chem Rev 113:6621-6658

2. Qu Y, Duan X (2013) Chem Soc Rev 42:2568-2580

3. Sousa C, Tosoni S, Illas F (2013) Chem Rev 113:4456-4495

4. Akimov AV, Neukirch AJ, Prezhdo OV (2013) Chem Rev 113:4496-4565

5. Szalay PG, Müller T, Gidofalvi G, Lischka H, Shepard R (2012) Chem Rev 112:108-181

6. Lyakh DI, Musia M, Lotrich VF, Bartlett RJ (2012) Chem Rev 112:182-243

7. Austin BM, Zubarev DY, Lester WA (2012) Chem Rev 112:263-288

8. Cohen AJ, Mori-Sanchez A, Yang W (2012) Chem Rev 112:289-320

9. Nørskov J, Abild-Pedersen F, Studt F, Bligaard T (2011) PNAS 108:937-943

10. Kohn W, Sham LJ (1965) Phys Rev 140:1133

11. Perdew JP (2003) Kurth S. In: Fiolhais C, Nogueira F, Marques $\mathrm{M}$ (eds) A primer in density functional theory. Springer, Berlin, pp 1-55

12. Perdew JP, Chevary JA, Vosko SH, Jackson KA, Pederson MR, Singh DJ, Fiolhais C (1992) Phys Rev B 46:6671-6687
13. Perdew JP, Burke K, Ernzerhof M (1996) Phys Rev Lett 77:3865-3868

14. Hammer B, Hansen LB, Nørskov JK (1999) Phys Rev B 59:7413-7421

15. Zhao Y, Truhlar DG (2005) J Phys Chem A 109:5656

16. Svelle S, Tuma C, Rozanska X, Kerber T, Sauer J (2009) J Am Chem Soc 131:816

17. Becke AD (1993) J. Chem. Phys. 98:5648-5652

18. Perdew JP, Wang Y (1986) Phys Rev B 33:8800

19. Perdew JP, Burke K, Ernzerhof M (1996) J Chem Phys 105:9982-9985

20. Adamo C, Barone V (1999) J Chem Phys 110:6158-6170

21. Ernzerhof M, Scuseria GE (1999) J. Chem. Phys. 110:5029-5036

22. Heyd J, Scuseria GE, Ernzerhof M (2003) J Chem Phys 118:8207-8215 ibid. (2006) 124:219906 (E)

23. Krukau AV, Vydrov OA, Izmaylov AF, Scuseria GE (2006) J Chem Phys 125:224106

24. Dovesi R, Saunders VR, Roetti C, Orlando R, Zicovich-Wilson CM, Pascale F, Civalleri B, Doll K, Harrison NM, Bush IJ, D'Arco Ph, Llunell M (2009) CRYSTAL09 user's manual. University of Torino, Torino

25. Paier J, Marsman M, Kresse G (2007) J Chem Phys 127:024103

26. Paier J, Marsman M, Hummer K, Kresse G, Gerber IC, Angyan JG (2006) J Chem Phys 124:154709 ibid. (2006) 125:249901 (E)

27. Todorova T, Seitsonen AP, Hutter J, Kuo IFW, Mundy CJ (2006) J Phys Chem B 110:3685-3691

28. Sauer J, Döbler J (2004) Dalton Trans 19:3116-3121

29. da Silva JLF, Ganduglia-Pirovano MV, Sauer J, Bayer V, Kresse G (2007) Phys Rev B 75:045121

30. Herzberg G (1989) Molecular spectra and molecular structure. I. Spectra of diatomic molecules, 2nd edn. Robert E. Krieger Publishing Co., Inc, Florida

31. Hubbard J (1964) Proc R Soc London, Ser A 276:238-257

32. Hubbard J (1964) Proc R Soc London, Ser. A 277:237-259

33. Hubbard J (1964) Proc R Soc London, Ser A 281:401-419

34. Anisimov VI, Aryasetiawan F, Lichtenstein AI (1997) J Phys: Condens. Matter 9:767-808

35. Dudarev SL, Botton GA, Savrasov SY, Humphreys CJ, Sutton AP (1998) Phys Rev B 57:1505-1509

36. Hu Z, Metiu H (2011) J Phys Chem C 115:5841-5845

37. Marom N, Tkatchenko A, Rossi M, Gobre VV, Hod O, Scheffler M, Kronik L (2011) J Chem Theory Comput 7:3944-3951

38. Langreth DC, Lundqvist BI, Chakarova-Käck SD, Cooper VR, Dion M, Hyldgaard P, Kelkkanen A, Kleis J, Kong L, Li S, Moses PG, Murray E, Puzder A, Rydberg H, Schröder E, Thonhauser T (2009) J Phys: Condens Matter 21:084203

39. Xu X, Goddard WA III (2004) Proc Natl Acad Sci USA 101:2673-2677

40. Zhao Y, Truhlar DG (2008) Acc Chem Res 41:157-167

41. Grimme S (2006) J Chem Phys 124:034108

42. von Lilienfeld OA, Tavernelli I, Röthlisberger U, Sebastiani D (2004) Phys Rev Lett 93:153004

43. Grimme S (2006) J Comput Chem 27:1787-1799

44. Grimme S, Antony J, Ehrlich S, Krieg HJ (2010) Chem Phys 132:154104

45. Tkatchenko A, Scheffler M (2009) Phys Rev Lett 102:073005

46. Zhao Y, Truhlar DG (2008) Theor Chem Acc 120:215

47. Zhao Y, Truhlar DG (2008) J Phys Chem A 110:13126

48. Zhao Y, Truhlar DG (2006) J Chem Phys 125:194101

49. Sterrer M, Heyde M, Novicki M, Nilius N, Risse T, Rust HP, Pacchioni G, Freund HJ (2006) J Phys Chem B 110:46-49

50. Alkauskas A, Broqvist P, Pasquarello A (2011) Phys Stat Sol B 248:775-789

51. Szabo A, Ostlund NS (1996) Modern quantum chemistry. McMillan, New York 
52. Shavitt I, Bartlett RJ (2009) Many-body methods in chemistry and physics: MBPT and coupled-cluster theory. Cambridge University Press, London

53. Onida G, Reining L, Rubio A (2002) Rev Mod Phys 74:601-659

54. Salpeter EE, Bethe HA (1951) Phys Rev 84:1232-1242

55. Chen W, Pasquarello A (2012) Phys Rev B 86:035134

56. Chen W, Pasquarello A (2012) Phys Rev B 88:115104

57. Lany S, Zunger A (2008) Phys Rev B 78:235104

58. Van de Walle CG, Neugebauer J (2004) J Appl Phys 95:3851-3879

59. Gallino F, Pacchioni G, Di Valentin C (2010) J Chem Phys 133:144512

60. Strehlow WH, Cook EL (1973) J Phys Chem Ref Data 2:163-199

61. Skone JH, Govoni M, Galli G (2014) Phys Rev B 89:195112

62. Marques MAL, Vidal J, Oliveira MJT, Reining L, Botti S (2011) Phys Rev B 83:035119

63. Conesa J (2012) J Phys Chem C 116:18884

64. Migani A, Mowbray DJ, Iacomino A, Zhao J, Petek H, Rubio A (2013) J Am Chem Soc 135:11429-11432

65. Migani A, Mowbray DJ, Zhao J, Petek H, Rubio A (2014) J Chem Theory Comput 10:2103

66. Onishi H, Aruga T, Egawa C, Iwasawa Y (1988) Surf Sci 193:33-46

67. Onda K, Li B, Zhao J, Petek H (2005) Surf Sci 593:32-37

68. Borodin A, Reichling M (2011) Phys Chem Chem Phys 13:15442-15447

69. Shishkin M, Kresse G (2006) Phys Rev B 74:035101

70. van Schilfgaarde M, Kotani T, Faleev S (2006) Phys Rev Lett 96:226402

71. Shishkin M, Marsman M, Kresse G (2007) Phys Rev Lett 99:246403

72. Shishkin M, Kresse G (2007) Phys Rev B 75:235102

73. Mars P, van Krevelen P (1954) Chem Eng Sci 3:41

74. Tsuji H, Hattori H (2004) ChemPhysChem 5:733

75. Pacchioni G (2003) ChemPhysChem 4:1041

76. Pala RGS, Metiu H (2007) J Phys Chem C 111:8617

77. Chrétien S, Metiu H (2006) Catal Lett 107:143

78. Shapovalov V, Metiu H (2007) J Catal 245:205

79. McFarland EW, Metiu H (2013) Chem Rev 113:4391

80. Shao X, Prada S, Giordano L, Pacchioni G, Nilius N, Freund HJ (2011) Angew Chem Int Ed 50:11525-11527
81. Stavale F, Shao X, Nilius N, Freund HJ, Prada S, Giordano L, Pacchioni G (2012) J Am Chem Soc 134:11380-11383

82. Prada S, Giordano L, Pacchioni G (2014) J Phys: Condens Matter 26:315004

83. Cui Y, Shao X, Baldofski M, Sauer J, Nilius N, Freund HJ (2013) Angew Chem Int Ed 52:11385-11387

84. Prada S, Giordano L, Pacchioni G (2013) J Phys Chem C 117:9943-9951

85. Pajer J, Penschke C, Sauer J (2013) Chem Rev 113:3949-3985

86. Migani A, Vayssilov GN, Bromley ST, Illas F, Neyman KM (2010) J Mater Chem 20:10535

87. Fabris S, de Gironcoli S, Baroni S, Vicario G, Balducci G (2005) Phys. Rev. B 71:041102

88. Kresse G, Blaha P, Da Silva JLF, Ganduglia-Pirovano MV (2005) Phys Rev B 72:237101

89. Huang M, Fabris S (2008) J Phys Chem C 112:8643

90. Branda MM, Castellani NJ, Grau-Crespo R, de Leeuw NH, Hernandez NC, Sanz JF, Neyman KM, Illas F (2009) J Chem Phys 131:094702

91. Graciani J, Marquez AM, Plata JJ, Ortega Y, Hernandez NC, Meyer A, Zicovich-Wilson CM, Sanz JF (2011) J Chem Theory Comput 7:56

92. Wang Y, Cheng HP (2013) J Phys Chem C 117:2106

93. Suntivich J, Gasteiger HA, Yabuuchi N, Nakanishi H, Goodenough JB, Shao-Horn Y (2011) Nat Chem 3:546-550

94. Kotomin EA, Mastrikov YA, Heifets E, Maier J (2008) Phys Chem Chem Phys 10:4644-4649

95. Chen HAT, Raghunath P, Lin MC (2011) Langmuir 27:6787-6793

96. Janesko BG, Henderson TM, Scuseria GE (2009) Phys Chem Chem Phys 11:443-454

97. Getsoian AB, Bell AT (2013) J Phys Chem C 117:25562-25578

98. Kronik L, Tkatchenko A (2014) Acc. Chem. Res. (in press).

99. Man IC, Soriga SG, Parvulescu V (2014) Chem Phys Lett 604:38-45

100. Zhang YK, Yang WT (1998) Phys Rev Lett 80:890

101. Dion M, Rydberg H, Schroder E, Langreth DC, Lundqvist BI (2004) Phys Rev Lett 92:246401

102. Pacchioni G (2008) J Chem Phys 128:182505 